\title{
Temperature Correction Using Degenerate Modes for Cylindrical Cavity Perturbation Measurements
}

\author{
Michael Barter ${ }^{\circledR}$, Samuel Partridge ${ }^{(}$, Daniel R. Slocombe ${ }^{\circledR}$, and Adrian Porch ${ }^{\circledR}$
}

\begin{abstract}
Microwave cavity perturbation measurements are a useful way to analyze material properties. Temperature changes can be introduced during these measurements either intentionally or as a result of some other process. The microwave cavity itself also has a temperature-dependent response, which can affect the results. A common method to correct is to use another resonant mode separately to the measurement mode, which is not affected by the sample. Instead of using independent modes, this paper describes a method to use split degenerate $T M_{m 10}$ modes of cylindrical cavities. $T M_{m 10}$ consists of two modes with identical field patterns with a relative rotation between them and identical resonant frequencies. A strategically placed perturbation reduces the frequency of one of the $T M_{m 10}$ modes and affects the coupling of both modes by reconfiguring the fields. This can be used for temperature correction by placing a sample such that both modes are equally coupled. The lower frequency, the perturbed mode is used as a measurement mode. The higher mode is used as a reference for temperature correction as it is unaffected by the sample. This technique was verified by measuring the permittivity of pure water using an aluminum microwave cavity resonator at $3.96 \mathrm{GHz}$. The temperature was swept between $20^{\circ} \mathrm{C}$ and $60{ }^{\circ} \mathrm{C}$, and the results was verified against the literature.
\end{abstract}

Index Terms-Cavity resonators, dielectric characterization, microwave measurement, permittivity, perturbation methods.

\section{INTRODUCTION}

$\mathbf{T}$ HE microwave cavity perturbation technique is a commonly used method of defining electromagnetic properties of materials due to its noninvasive and nondestructive qualities [1]-[5]. This technique involves perturbing a resonant electromagnetic field with a sample and measuring the change in the frequency response. Many measurements carried out with the microwave perturbation technique involve a change in temperature such as environmental changes or exothermic/endothermic chemical reactions within the sample [6]-[9]. Alternatively, the change can be intentional while examining dielectric properties over a range of temperatures. As the measured resonant frequency and bandwidth of the cavity are a function of the cavity geometry, a method of decoupling changes due to cavity expansion/contraction from the changes in sample dielectric properties is essential.

Manuscript received June 20, 2018; revised September 17, 2018; accepted October 7, 2018. This work was supported in part by the EPSRC, in part by the STFC, and in part by Merck KGaA. (Corresponding author: Michael Barter.)

The authors are with the Centre for High Frequency Engineering, School of Engineering, Cardiff University, Cardiff CF24 3AA, U.K. (e-mail: barterm@cf.ac.uk; partridges1@cf.ac.uk; slocombed1@cf.ac.uk; porcha@cf.ac.uk).

Color versions of one or more of the figures in this paper are available online at http://ieeexplore.ieee.org.

Digital Object Identifier 10.1109/TMTT.2018.2882480
All cylindrical cavities have modes that can be considered degenerate. Degenerate modes occur when the two cavity modes have the same resonant frequency. Mode degeneracy can have two different causes. The roots of Bessel functions that define two different modes can force the frequencies to be equal, for example, cylindrical modes $\mathrm{TM}_{111}$ and $\mathrm{TE}_{011}$ [since $J_{0}^{\prime}(x)=-J_{1}(x)$ ] [10]. Alternatively, degenerate modes can occur when the rotational symmetry of the mode allows different possible orientations such as cylindrical mode $\mathrm{TM}_{m 10}$ (where $m \geq 1$ ) [10].

Assuming a perfectly constructed microwave cavity, these degenerate modes would have exactly the same resonant frequency. In practice, inevitable small imperfections in the cavity construction can cause these modes to have slightly different resonant frequencies. By inserting a sample away from the central axis of the cavity, it is possible to separate the resonant frequencies of the modes. This is often referred to as a mode trap [7], [11]. Perturbing the cavity fields causes the orientation of one of the possible modes to align with a local maximum at the sample. The other mode would have a local minimum. This sample can be placed at a location to suppress the degenerate mode, by ensuring that it is not coupled to. Alternatively, the sample can be placed at a location such that both orientations are equally coupled. Hence it is possible to use equally coupled, split degenerate modes to correct for changes in temperature during perturbation measurements. The main advantage of the proposed method is the much narrower bandwidth compared with using a nondegenerate reference mode. This is described in Section III-A, for example, $\mathrm{TM}_{310}$ at $6.62 \mathrm{GHz}$ can be used as a reference for measurements using $\mathrm{TM}_{010}$ at $2.50 \mathrm{GHz}$, leading to a wide bandwidth. This proposed method dramatically simplifies the instrumentation for dielectric sensor applications, where a measurement bandwidth of less than $100 \mathrm{MHz}$ will suffice. A further advantage is a reduction in other systematic errors, such as variations in the coupling coefficients as a function of frequency.

\section{Splitting the Degeneracy of $\mathrm{TM}_{m} 10$ Modes}

$\mathrm{TM}_{m n p}$ modes are described by the integers $m, n$, and $p$, where $m$ is the azimuthal periodicity, $n$ is the number of Bessel function maxima between the axis and circumference, and $p$ is the number of nodes in the $z$-direction. The axial electric field amplitude $\left(E_{z}\right)$ is described by (1) derived from [12]

$$
E_{z}(r, \phi, z)=E_{0} J_{m}\left(\frac{k_{m n} r}{a}\right) \cos (m(\phi+\theta)) \cos \left(\frac{p \pi z}{l}\right)
$$




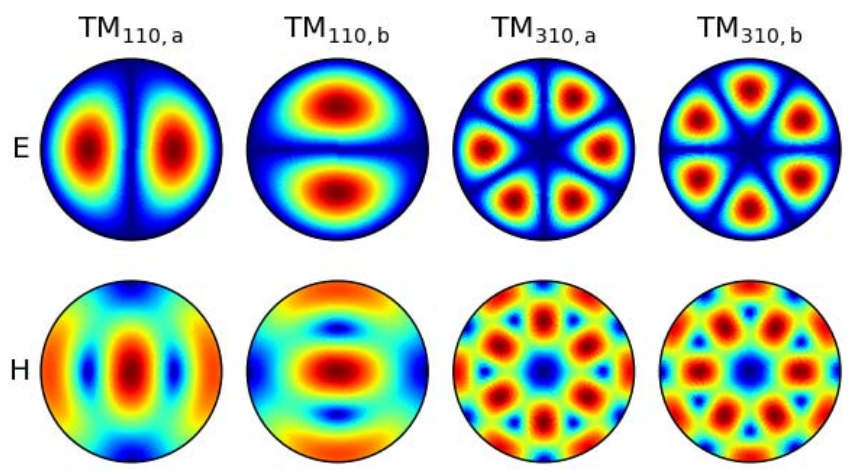

Fig. 1. Electric field (E) and magnetic field $(\mathrm{H})$ patterns for the degenerate $\mathrm{TM}_{110, a}, \mathrm{TM}_{110, b}, \mathrm{TM}_{310, a}$, and $\mathrm{TM}_{310, b}$ modes.
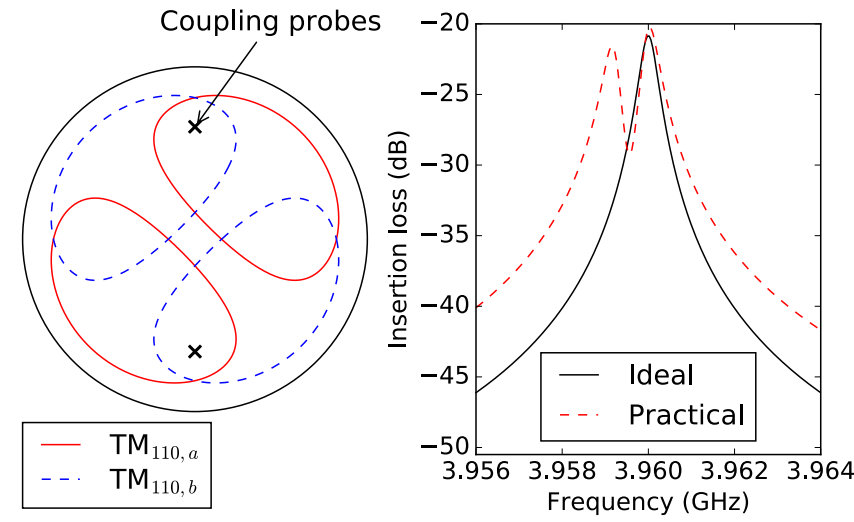

Fig. 2. Schematic contour plot of the electric field magnitude for the $\mathrm{TM}_{110}$ mode with capacitive (i.e., E-field) coupling probes, located as shown for a transmission measurement (left). Measured transmitted power $\left|S_{21}\right|^{2}$ in the frequency domain of the $\mathrm{TM}_{110}$ mode in a cavity split in the vertical plane in the ideal case (black solid line) and in the practical case (red dashed line).

where $a$ is the cavity radius, $l$ is the cavity height, $\theta$ is the axial rotation (resulting in the $\mathrm{TM}_{m 10, a}$ and $\mathrm{TM}_{m 10, b}$ variants), and $k_{m n}$ is the $n$th root of the $m$ th-order Bessel function of the first kind. The field patterns of these modes are shown in Fig. 1.

With a perfectly symmetrical cylindrical microwave cavity, the spatial orientations of the $\mathrm{TM}_{m 10}$ modes result in exactly the same frequency, thus yielding a single Lorentzian response. Distortion or splitting of the Lorentzian response can occur when the frequencies are unequal. This can happen when the azimuthal symmetry of the cylindrical cavity is broken, such as with a split in the cavity construction in the vertical plane, or where there are holes in the cavity. These ideal and practical scenarios can be seen in Fig. 2 along with the orientation of $\mathrm{TM}_{110, a}$ and $\mathrm{TM}_{110, b}$ modes.

In the practical scenario, it is difficult to extract Lorentzian curve parameters from the $\mathrm{TM}_{110}$ mode due to the distortion of the Lorentzian peak. A perturbation can be positioned to maintain equal coupling between the two modes while also increasing separation between the $\mathrm{TM}_{110, a}$ and $\mathrm{TM}_{110, b}$ modes. If the perturbation is large enough to ensure sufficient separation, then the unperturbed $\mathrm{TM}_{110}$ mode can be used for temperature correction. This is discussed in Section III-B.

\section{TEMPERATURE CORRECTION USING SPLit DEGENERATE MODES}

Many microwave cavity perturbation measurements involve a change in temperature of the measurement cavity. Regardless of the reason behind it, a change in cavity temperature causes several changes to the host cavity that affect the frequency response of the system; change in cavity geometry, coupling geometry, and temperature-dependent electrical conductivity of the cavity walls. These temperature changes during microwave perturbation measurements cause changes in measured frequency. This must be accounted for so that any changes in the resonant frequency are caused by changes in the sample.

\section{A. Method of Temperature Correction and Measurement}

Previously, temperature correction has been carried out using two-independent modes [13], [14]. One mode is used to measure the sample, most frequently an axially placed sample measured using one of the $\mathrm{TM}_{0 n 0}$ modes. This mode is chosen as the electric field and can be assumed to be constant over the sample volume with a high electric field and negligible magnetic field at the location of the sample. A second mode is chosen which has no electric or magnetic field at the location of the sample. For axially placed samples, this is often one of the $\mathrm{TM}_{m 10}$ modes, where $m \geq 1$. The sample will have no effect on the frequency response of this mode, meaning measured changes can be assumed to be from the temperature response of the cavity. These choices allow the measurement mode to be sensitive to the sample while the reference mode allows for temperature correction.

Using the microwave characterization technique, the measured resonant frequency $f_{0}$ can be calculated using the following:

$$
f_{0}=\frac{c}{2 \pi} \sqrt{\left(\frac{k_{m n}}{a(T)}\right)^{2}+\left(\frac{p \pi}{l(T)}\right)^{2}}
$$

where $c$ is the speed of light, $n, m$, and $p$ are the mode integers, $k_{m n}$ is the $n$th root of the $m$ th-order Bessel function of the first kind, and $a(T)$ and $l(T)$ are the temperature-dependent radius and height of the cavity, respectively. As the cavity undergoes a change in temperature, this causes a change in the geometry of the cavity. As described in [13] and [14], by using first-order partial derivatives, the changes in resonant frequency due to a change in temperature can be seen as follows:

$$
\frac{\Delta f_{0}}{f_{0}} \approx-\alpha_{c} \Delta T
$$

where $\Delta T$ is the change in temperature and $\alpha_{c}$ is the linear thermal expansion coefficient. This equation only accounts for changes in the cavity geometry-the main source of temperature-dependent error.

Equation (4) is used to correct the frequency measurements for temperature changes [13], [14]

$$
\begin{aligned}
\frac{\Delta f_{s}(T)}{f_{s}(0)} \approx\left[\frac{\Delta f_{010, s}(T)}{f_{010, s}(0)}\right. & \left.-\frac{\Delta f_{m 10, s}(T)}{f_{m 10, s}(0)}\right] \\
- & {\left[\frac{\Delta f_{010, u}(T)}{f_{010, u}(0)}-\frac{\Delta f_{m 10, u}(T)}{f_{m 10, u}(0)}\right] }
\end{aligned}
$$


where $u$ represents the unperturbed cavity and $s$ when the sample is present. As (3) suggests, any change in temperature causes a proportional change in resonant frequency of the cavity. This change in frequency has the same fractional shift for an ideal cavity regardless of cavity mode. When the sample is present, the frequency can be corrected by subtracting the fractional frequency shift of the reference mode from the fractional frequency shift of the measurement mode. This can be seen in the sample related terms in (4). The unperturbed terms of this equation are included to remove any errors caused by differences in gradient from the thermal expansion coefficient. These differences are caused by imperfections in the cavity construction and material.

With corrected frequency measurements, the values for real permittivity can then be calculated using the following:

$$
\varepsilon_{1}(T) \approx-2\left[\frac{f_{s}(T)-f_{0}(0)}{f_{0}(0)}\right] \frac{V_{c}}{V_{s}} G_{m n p}+1
$$

where $V_{c}$ and $V_{s}$ are the cavity and sample volumes, respectively. The dimensionless geometry factor $G_{m n p}$ can be calculated by placing a known sample into the cavity, such as a quartz rod, and verified by simulation (e.g., COMSOL) or through analytical calculations. As this method uses an intermodal relationship between cavity modes, no previous measurements of expansion coefficients are required [13], [14].

\section{B. Proposed Use of Degenerate Modes}

Degenerate modes, as discussed in Section II, can be used as an alternative method for correcting the temperature changes. This method uses the same analysis, as discussed in Section III-A. When a sample is inserted into the cavity offaxis, this causes degenerate modes to split. The placement of the sample must be chosen to ensure both modes are equally coupled. For modes $\mathrm{TM}_{110}$ and $\mathrm{TM}_{310}$ used in this paper, the sample is inserted at an angle of $45^{\circ}$ from the coupling structures, however, this results in $\mathrm{TM}_{210}$ and $\mathrm{TM}_{410}$ being unusable. This is shown in Fig. 3.

This perturbation locks the orientation of the modes. One of the modes will be perturbed by the sample and thus will have a lower frequency. This lower frequency mode becomes the measurement mode and is referred to as $\mathrm{TM}_{m 10, a}$. The higher frequency of the two modes will not be affected by the introduction of the perturbing sample. This unaffected mode becomes the reference mode and is used for temperature corrections. This mode is referred to as $\mathrm{TM}_{m 10, b}$.

Due to the geometry of the modes, not all of them can be coupled equally at once. Placing the sample at $45^{\circ}$ from the coupling probes allows the use of the $\mathrm{TM}_{110}$ and $\mathrm{TM}_{310}$ modes. $\mathrm{TM}_{210}$ and $\mathrm{TM}_{410}$ are unusable due to only coupling the unperturbed and perturbed modes, respectively. The technique used in Section III-A is utilized to correct for temperature while replacing $\mathrm{TM}_{010}$ and $\mathrm{TM}_{m 10}$ modes in (4), with $\mathrm{TM}_{m 10, a}$ and $\mathrm{TM}_{m 10, b}$ modes, respectively.
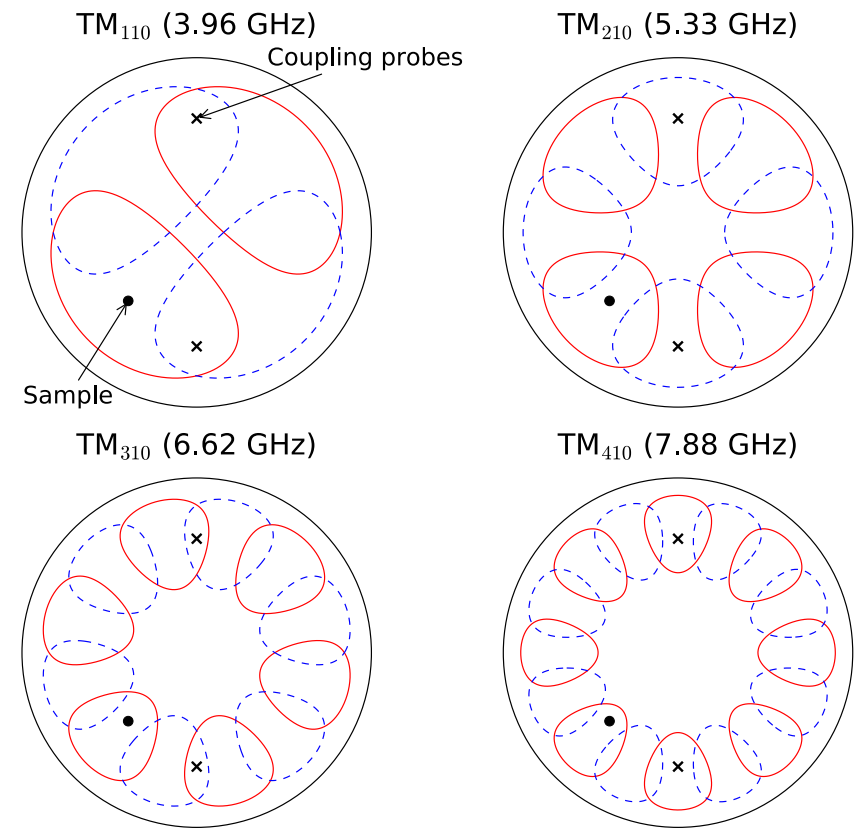

Fig. 3. Contour plots of $\mathrm{TM}_{m 10}$ modes. $\mathrm{TM}_{110}$ and $\mathrm{TM}_{310}$ have equal coupling between the perturbed and unperturbed modes. Only the unperturbed $\mathrm{TM}_{210}$ mode is coupled, and only the perturbed $\mathrm{TM}_{410}$ mode is coupled.

Using this technique, the sample is placed in the region of the high electric field and notionally zero magnetic field of the measurement mode $\mathrm{TM}_{m 10, a}$. When considering the fields of the reference mode $\mathrm{TM}_{m 10, b}$, the sample is in a region of the low electric field but a high magnetic field. As described in Section III-A, in order for this technique to work for any material, the reference mode must have no magnetic of the electric field at the sample location. Due to this the degenerate mode, temperature correction technique described may only be used on the samples, which are nonmagnetic.

\section{Method And Results}

These experiments were conducted using an aluminum microwave cavity resonator designed for TM measurements with capacitive antenna coupling into the E-field. Measurements were taken using the $\mathrm{TM}_{110}$ mode configuration shown in Fig. 3. The $\mathrm{TM}_{310}$ mode was not used due to interference from another mode with similar resonant frequency.

The temperature was controlled by placing the resonant cavity in a Memmert IPP400 temperature controlled oven. The transmission $\left(S_{21}\right)$ measurements were obtained using Keysight Technologies Vector Network Analyzer (VNA) (PNA-L N5232A) with RF cables from Huber+Suhner. The VNA was connected to a PC running an in-house measurement acquisition program built with LabVIEW. The program also measures the temperature of the cavity using an Omega PT100 temperature sensor connected to a National Instruments (NI) cDAQ-9171. This is shown in Fig. 4.

The experiment consisted of two temperature ramp measurements, one with empty quartz tubes and the other with the addition of a water sample. Two nested quartz tubes were used. The outer tube has an outer diameter of $4.0 \mathrm{~mm}$ 


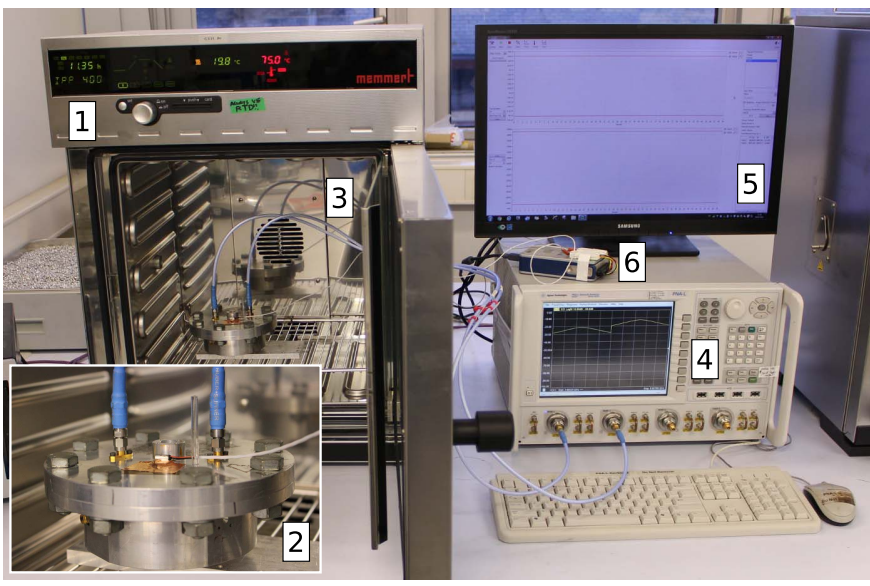

Fig. 4. (1) Experimental setup showing the temperature controlled oven (2) containing the microwave cavity resonator with the inserted sample and attached temperature sensor. (3) Coaxial cables (4) connect the cavity to the VNA, which is being driven by (5) LabVIEW measurement acquisition program. (6) Temperature sensor is connected to the NI-DAQ, also controlled by the same program.

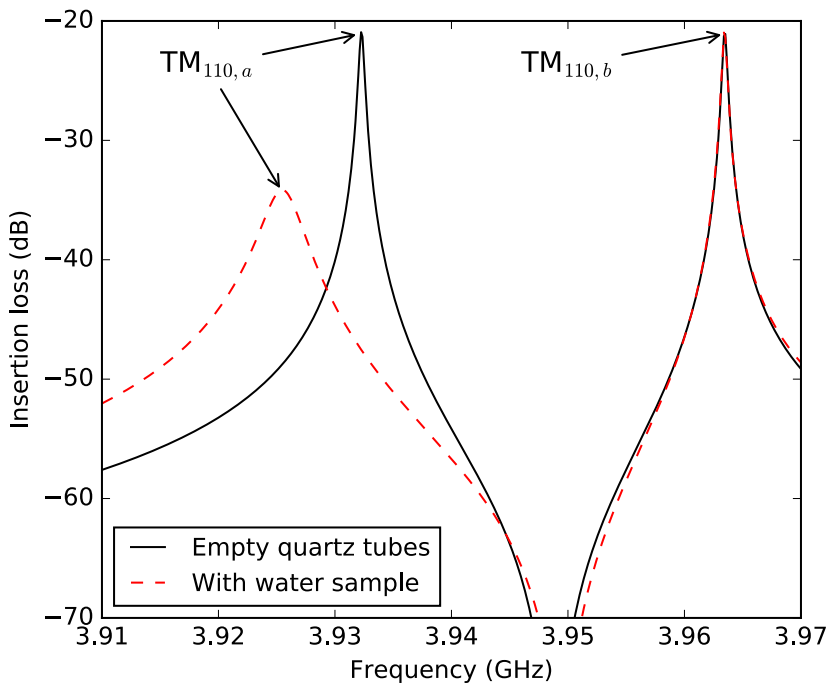

Fig. 5. Measured transmitted power $\left|S_{21}\right|^{2}$ in the frequency domain of the $\mathrm{TM}_{110}$ mode when perturbed by the empty quartz tubes (black solid line) and when a water sample is added (red dashed line).

and the inner diameter of $2.0 \mathrm{~mm}$. The inner tube has an outer diameter of $0.4 \mathrm{~mm}$ and the inner diameter of $0.3 \mathrm{~mm}$. The purpose of the outer tube was to split the high and low $\mathrm{TM}_{110}$ modes enough to treat them as separate modes and reliably extract the frequency and $Q$-factor. The inner tube is used as a container for a small volume of water. The frequency response showing the separation of these modes can be seen with and without the water sample in Fig. 5.

The temperature ramps were performed from $20^{\circ} \mathrm{C}$ to $60^{\circ} \mathrm{C}$ over $8 \mathrm{~h}$, then returning to $20{ }^{\circ} \mathrm{C}$ at the same rate. Both the measurement mode $\mathrm{TM}_{110, a}$ and reference mode $\mathrm{TM}_{110, b}$ were measured for the empty and water ramps. The frequency results of these measurements are shown in Fig. 6.

Frequency results show the reference mode $\mathrm{TM}_{110, b}$ is not affected by the presence of the sample, as described

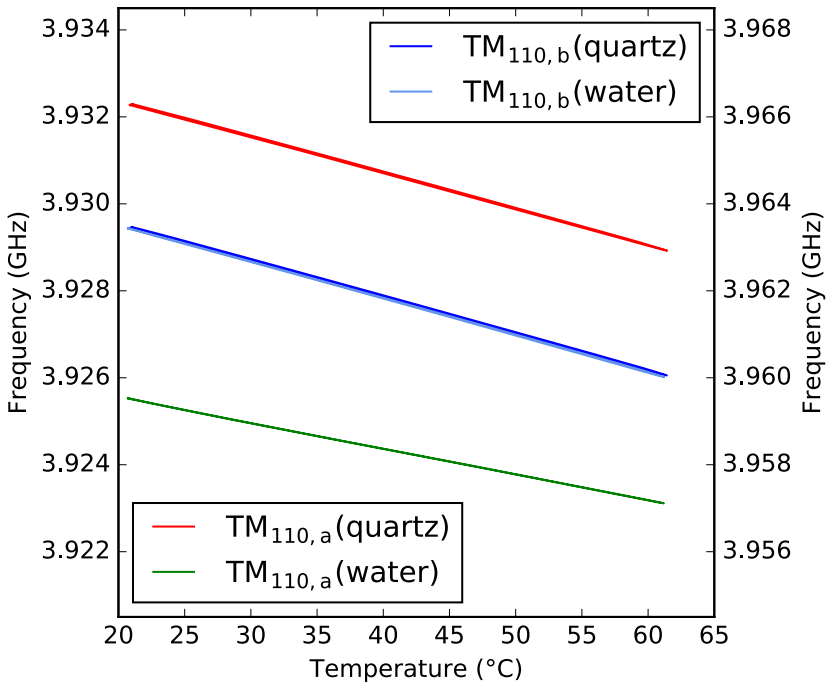

Fig. 6. $\mathrm{TM}_{110}$ measured frequency versus temperature with measurement modes on the left axis and reference modes on the right axis.

in Section III-B. The measurements for the $\mathrm{TM}_{110, a}$ mode have different resonant frequencies for water and quartz due to the addition of water affecting the perturbation. These measurement modes also have different gradients. The gradient in the quartz tubes is caused predominantly by the change in cavity temperature as the permittivity of quartz has very little temperature dependence. The gradient of the water containing sample has the same contribution from the temperature change of the cavity and quartz, but also a contribution due to the temperature dependence of permittivity for water. Before temperature correction of the water sample, the frequency decreases as the temperature increases. This trend is the opposite of the expected result. To resolve this, the temperature correction technique explained in Section III-B is used to remove the contributions from the cavity and quartz tubes, as well as reduce the errors present. This method ensures that after correcting these values, the only changes in observed frequency are due to changes in the permittivity of water. The real permittivity of water is calculated using (5) before and after temperature correction of frequency has been carried out. This can be seen in Fig. 7.

Real permittivity values shown in Fig. 7 show that before temperature correction the graph suggests a rise in permittivity of the water sample as temperature increases. Once this data has been corrected, a decrease in real permittivity can be seen between $20{ }^{\circ} \mathrm{C}$ and $60{ }^{\circ} \mathrm{C}$. This corrected negative gradient and corresponding $\varepsilon_{1}$ values now closely match the literature values calculated from the equations developed by Kaatze [15]. This also matches closely with the values seen in the literature [16], [17]. The errors for these measurements were also calculated and compared with the literature values, as shown in Table I.

The main systematic errors accounted for include the permittivity and volume of the calibration quartz rod, and the volume of the water sample. These are approximately $2 \%$. The random errors were negligible in comparison, less than $0.1 \%$. As the table shows, the experimental results were well within 


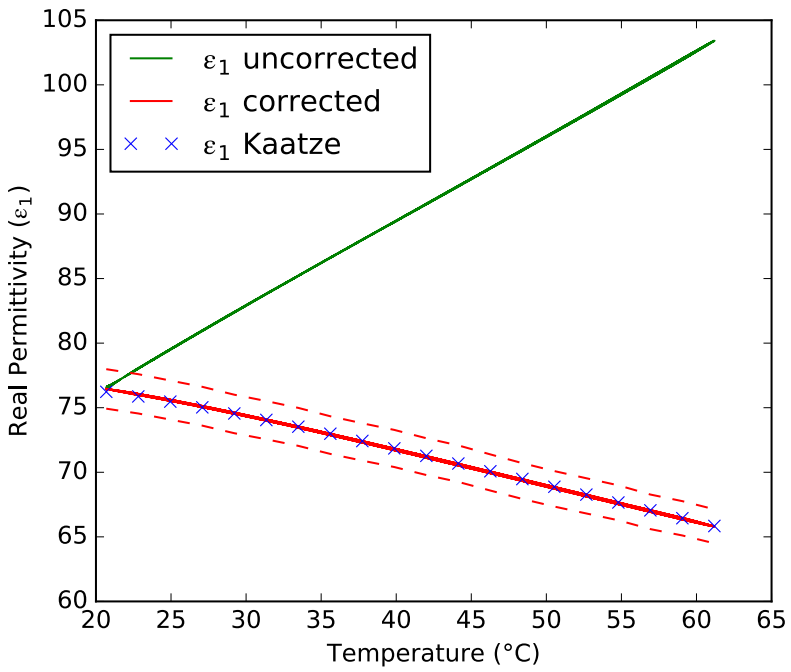

Fig. 7. Corrected, uncorrected, and literature values for real permittivity $\left(\varepsilon_{1}\right)$ of water at $4 \mathrm{GHz}$ versus temperature with $2 \%$ systematic error (dotted lines).

\section{TABLE I}

COMPARISON OF EXPERIMENTAL MEASUREMENTS Versus Literature VAlues of The PermitTivity OF WATER AT $4 \mathrm{GHz}$

\begin{tabular}{|c|c|c|c|}
\hline $\mathrm{T}\left({ }^{\circ} \mathrm{C}\right)$ & $\varepsilon_{1, \text { Kaatze }}$ & $\varepsilon_{1, \text { measured }}$ & Percentage difference \\
\hline 25.0 & 75.46 & 75.54 & $0.11 \%$ \\
\hline 30.0 & 74.37 & 74.43 & $0.08 \%$ \\
\hline 35.0 & 73.14 & 73.04 & $-0.13 \%$ \\
\hline 40.0 & 71.82 & 71.67 & $-0.21 \%$ \\
\hline 45.0 & 70.44 & 70.27 & $-0.24 \%$ \\
\hline 50.0 & 69.03 & 68.91 & $-0.17 \%$ \\
\hline 55.0 & 67.60 & 67.49 & $-0.17 \%$ \\
\hline 60.0 & 66.18 & 66.18 & $0.01 \%$ \\
\hline
\end{tabular}

the systematic error when compared against the literature permittivity values.

\section{Conclusion}

Temperature correction was carried out on cavity perturbation measurements by splitting degenerate modes and using one as a robust and precise reference. This paper shows that degenerate $\mathrm{TM}_{m 10}$ modes can be reconfigured by perturbing the cavity. This is useful to prevent the interference between the higher and lower frequency $\mathrm{TM}_{m 10}$ modes. The position of the mode relative to the cavity coupling structures determines how strongly that mode is coupled.

The use of $\mathrm{TM}_{m 10}$ modes is applied to the temperature correction technique developed by Cuenca et al. [13]. This technique is required due to the temperature-dependent properties of the microwave cavity. The sample is placed in a position to ensure equal coupling of both $\mathrm{TM}_{m 10}$ modes. The lower frequency perturbed mode is used to measure the sample, and the higher mode is used as a reference unaffected by the sample.

To verify this technique, the temperature-dependent properties of the water were investigated. Before correction, the frequency response of the water sample appears to be incorrect due to the dominating temperature-dependent properties of the microwave cavity. After temperature correction, the values were verified against the literature and confirmed to be accurate.

\section{ACKNOWLEDGMENT}

Information about the data underpinning the results presented here, including how to access them, can be found in the Cardiff University Data Catalogue at http://doi.org/10.17035/d.2018.0050743125.

\section{REFERENCES}

[1] A. Porch et al., "Microwave treatment in oil refining," Appl. Petrochem. Res., vol. 2, nos. 1-2, pp. 37-44, Sep. 2012.

[2] J. Sheen, "Amendment of cavity perturbation technique for loss tangent measurement at microwave frequencies," J. Appl. Phys., vol. 102, no. 1, pp. 1-6, Apr. 2007.

[3] K. T. Mathew and U. Raveendranath, "Cavity perturbation techniques for measuring dielectric parameters of water and other allied liquids," Sens. Update, vol. 7, no. 1, pp. 185-210, Jan. 2000.

[4] H. Choi, J. Cuenca, G. Attard, and A. Porch, "A novel concentration detection method of hydrogen peroxide using microwave cavity perturbation technique," in Proc. 44th Eur. Microw. Conf., Oct. 2014, pp. 632-635.

[5] K. Sudheendran, D. Pamu, M. G. Krishna, and K. C. J. Raju, "Determination of dielectric constant and loss of high-K thin films in the microwave frequencies," Measurement, vol. 43, no. 4, pp. 556-562, May 2010.

[6] M. Dietrich, D. Rauch, A. Porch, and R. Moos, "A laboratory test setup for in situ measurements of the dielectric properties of catalyst powder samples under reaction conditions by microwave cavity perturbation: Set up and initial tests," Sensors, vol. 14, no. 9, p. 16856-16868, Sep. 2014

[7] N. Clark, N. Jones, and A. Porch, "Measurement of average particle size in metal powders by microwave cavity perturbation in the magnetic field," Sens. Actuators A, Phys., vol. 259, pp. 137-143, Jun. 2017.

[8] J. Hartley, A. Porch, and M. Jones, "A non-invasive microwave method for assessing solid-state ammonia storage," Sens. Actuators B, Chem., vol. 210, pp. 726-730, Apr. 2015.

[9] J. Krupka, K. Derzakowski, B. Riddle, and J. Baker-Jarvis, "A dielectric resonator for measurements of complex permittivity of low loss dielectric materials as a function of temperature," Meas. Sci. Technol., vol. 9, no. 10, pp. 1751-1756, Oct. 1999.

[10] R. N. Clarke et al.,"A guide to the characterisation of dielectric materials at RF and microwave frequencies," Inst. Meas. Control, London, U.K., Tech. Rep., 2003.

[11] T. Hanaguri, K. Takaki, Y. Tsuchiya, and A. Maeda, "An instrument for low- and variable-temperature millimeter-wave surface impedance measurements under magnetic fields," Rev. Sci. Instrum., vol. 74, no. 10, pp. 4436-4441, Oct. 2003.

[12] D. M. Pozar, Microwave Engineering, 4th ed. Hoboken, NJ, USA: Wiley, 2005.

[13] J. A. Cuenca, D. R. Slocombe, and A. Porch, "Temperature correction for cylindrical cavity perturbation measurements," IEEE Trans. Microw. Theory Techn., vol. 65, no. 6, pp. 2153-2161, Jun. 2017.

[14] J. A. Cuenca, D. R. Slocombe, and A. Porch, "Corrections to 'Temperature correction for cylindrical cavity perturbation measurements' [Jun 17 2153-2161]," IEEE Trans. Microw. Theory Techn., vol. 65, no. 12 , p. 5078 , Dec. 2017

[15] U. Kaatze, "Complex permittivity of water as a function of frequency and temperature," J. Chem. Eng. Data, vol. 34, no. 4, pp. 371-374, Oct. 1989

[16] W. J. Ellison, "Permittivity of pure water, at standard atmospheric pressure, over the frequency range $0-25 \mathrm{THz}$ and the temperature range 0-100 ${ }^{\circ}$ C, J. Jhys. Chem. Reference Data, vol. 36, no. 1, pp. 1-18, Feb. 2007.

[17] M. Merabet and T. K. Bose, "Dielectric measurements of water in the radio and microwave frequencies by time domain reflectometry," J. Phys. Chem., vol. 92, no. 21, pp. 6149-6150, Oct. 1988 


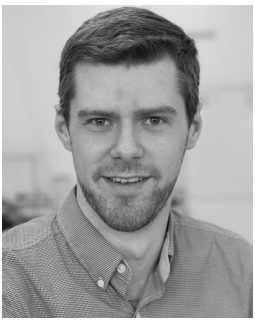

Michael Barter received the B.Eng. degree in electrical and electronic engineering from Cardiff University, Cardiff, U.K., in 2015, where he is currently pursuing the Ph.D. degree at the Centre for High Frequency Engineering.

$\mathrm{He}$ is currently sponsored by The Engineering and Physical Sciences Research Council and The Science and Technology Facilities Council. His current research interests include simultaneous neutron diffraction and microwave characterization of solid state ammonia storage materials, including metal-organic frameworks and inorganic salts. The overall aim of his research is to investigate novel ammonia storage materials for energy storage applications.

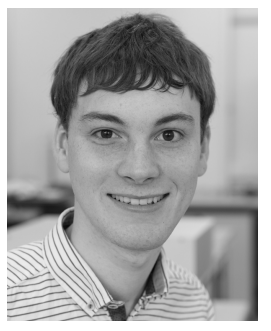

Samuel Partridge received the B.Eng. degree in electrical and electronic engineering from Cardiff University, Cardiff, U.K., in 2015, where he is currently pursuing the Ph.D. degree at the Centre for High Frequency Engineering.

$\mathrm{He}$ is currently sponsored by The Engineering and Physical Sciences Research Council and Merck $\mathrm{KGaA}$. His current research interests include the microwave characterization of functional pigments during exposure to external environmental conditions with the aim of understanding how these pigments are likely to respond in real-world applications.

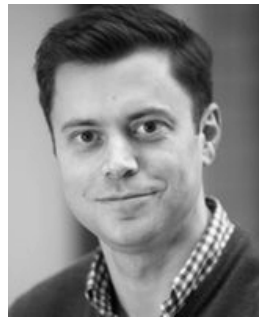

Daniel R. Slocombe received the Ph.D. degree in electronic engineering from Cardiff University, Cardiff, U.K., in 2012.

From 2002 to 2006, he was an Engineer with the Royal Air Force, where he was involved in airborne radar systems. He was a Research Fellow with the Inorganic Chemistry Laboratory, University of Oxford, Oxford, U.K., from 2012 to 2015 . He is currently a Lecturer with the School of Engineering, Cardiff University, where he is a member of the Centre for High Frequency Engineering. His current research interests include microwave science and materials, including microwave activation of catalytic processes, synthesis of functional oxides, dielectric spectroscopy, and new methods using magnetic resonance phenomena.

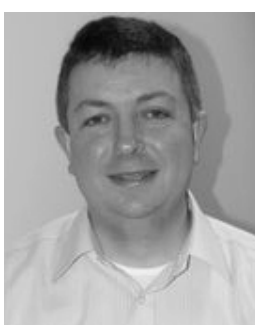

Adrian Porch received the M.A. degree in physics and the Ph.D. degree in low-temperature physics from Cambridge University, Cambridge, U.K., in 1989.

$\mathrm{He}$ is currently a Professor with the School of Engineering, Cardiff University, Cardiff, U.K., where he is a Research Leader with the Centre for High Frequency Engineering. He has 30 years of experience in applying microwave methods to measure and understand the fundamental properties of electronic materials. More recently, his techniques have been used to develop new types of electromagnetic sensors with an emphasis on applications across different disciplines. 